\title{
Indestructibility, strongness, and level by level equivalence
}

\author{
by
}

\author{
Arthur W. Apter (New York, NY)
}

\begin{abstract}
We construct a model in which there is a strong cardinal $\kappa$ whose strongness is indestructible under $\kappa$-strategically closed forcing and in which level by level equivalence between strong compactness and supercompactness holds non-trivially.
\end{abstract}

1. Introduction and preliminaries. A very surprising fact (see Theorems $5-7$ of [3]) is that if there are large enough cardinals in the universe, then indestructibility for either a strong or supercompact cardinal (in the sense of [5] or [13]) is incompatible with level by level equivalence between strong compactness and supercompactness. Indeed, Theorem 6 of [3] states that if $\kappa$ is a strong cardinal such that forcing with any $\kappa$-strategically closed partial ordering preserves $\kappa$ 's strongness (where for the rest of this paper, we will refer to such a cardinal as an indestructible strong cardinal) and level by level equivalence between strong compactness and supercompactness holds below $\kappa$ (where for the rest of this paper, level by level equivalence between strong compactness and supercompactness means that for $\delta \leq \lambda, \delta$ and $\lambda$ both regular, $\delta$ is $\lambda$ strongly compact iff $\delta$ is $\lambda$ supercompact), then no cardinal $\lambda>\kappa$ is $2^{\lambda}$ supercompact. However, a question left unanswered in [3] is whether it is possible for there to be level by level equivalence non-trivially below an indestructible strong cardinal $\kappa$ in a universe in which no cardinal $\lambda>\kappa$ is $2^{\lambda}$ supercompact. By the next to last sentence, the existence of such a universe is the best possible outcome for which one could hope.

The purpose of this paper is to provide an affirmative answer to the aforementioned question. Specifically, we prove the following theorem.

2000 Mathematics Subject Classification: 03E35, 03E55.

Key words and phrases: supercompact cardinal, strongly compact cardinal, strong cardinal, level by level equivalence between strong compactness and supercompactness.

The author wishes to thank Joel Hamkins for helpful conversations on the subject matter of this paper. The author also wishes to thank the referee for helpful comments and suggestions that have been incorporated into this version of the paper. 
THEOREM 1. Suppose $V_{0} \vDash " Z F C+\kappa_{0}$ is supercompact + There are no cardinals $\delta<\lambda$ such that $\delta$ is $\lambda$ supercompact and $\lambda$ is measurable". There is then a cardinal $\kappa<\kappa_{0}$, a submodel $V$ of a (possibly trivial) forcing extension of $V_{0}$, and a partial ordering $\mathbb{P} \in V$ such that $V^{\mathbb{P}} \vDash " Z F C+N o$ cardinal $\lambda>\kappa$ is $2^{\lambda}$ supercompact $+\kappa$ is an indestructible strong cardinal + For any $\gamma<\kappa$, there is a cardinal $\delta \in[\gamma, \kappa)$ which is $\delta^{+\gamma}$ supercompact + Level by level equivalence between strong compactness and supercompactness holds".

It will in fact be the case, roughly speaking, that however much supercompactness $\kappa_{0}$ reflects will also occur unboundedly often below $\kappa$ in $V_{0}, V$, and $V^{\mathbb{P}}$. Thus, e.g., $\left\{\delta<\kappa: \delta\right.$ is $\delta^{+\delta}$ supercompact $\}$ is unbounded in $\kappa$ in these models. We will comment again on this both at the beginning and at the conclusion of the proof of Theorem 1.

Before beginning the proof of Theorem 1, we briefly mention some preliminary information. Essentially, our notation and terminology are standard, and when this is not the case, this will be clearly noted. For $\alpha<\beta$ ordinals, $[\alpha, \beta],[\alpha, \beta),(\alpha, \beta]$, and $(\alpha, \beta)$ are as in standard interval notation.

When forcing, $q \geq p$ will mean that $q$ is stronger than $p$. If $G$ is $V$-generic over $\mathbb{P}$, we will use both $V[G]$ and $V^{\mathbb{P}}$ to indicate the universe obtained by forcing with $\mathbb{P}$. If $x \in V[G]$, then $\dot{x}$ will be a term in $V$ for $x$. We may, from time to time, confuse terms with the sets they denote and write $x$ when we actually mean $\dot{x}$, especially when $x$ is some variant of the generic set $G$, or $x$ is in the ground model $V$.

If $\kappa$ is a regular cardinal and $\mathbb{P}$ is a partial ordering, $\mathbb{P}$ is $\kappa$-distributive if for every sequence $\left\langle D_{\alpha}: \alpha<\kappa\right\rangle$ of dense open subsets of $\mathbb{P}, \bigcap_{\alpha<\kappa} D_{\alpha}$ is dense open. $\mathbb{P}$ is $\kappa$-strategically closed if in the two-person game in which the players construct an increasing sequence $\left\langle p_{\alpha}: \alpha \leq \kappa\right\rangle$, where player I plays odd stages and player II plays even and limit stages (choosing the trivial condition at stage 0 ), player II has a strategy which ensures the game can always be continued. Note that if $\mathbb{P}$ is $\kappa$-strategically closed, then $\mathbb{P}$ is $\kappa$-distributive. Also, if $\mathbb{P}$ is $\kappa$-distributive and $f: \kappa \rightarrow V$ is a function in $V^{\mathbb{P}}$, then $f \in V$. $\mathbb{P}$ is $\prec \kappa$-strategically closed if in the two-person game in which the players construct an increasing sequence $\left\langle p_{\alpha}: \alpha<\kappa\right\rangle$, where player I plays odd stages and player II plays even and limit stages (again choosing the trivial condition at stage 0 ), player II has a strategy which ensures the game can always be continued.

We recall for the benefit of readers Hamkins' definition from Section 3 of [10] of the lottery sum of a collection of partial orderings. If $\mathfrak{A}$ is a collection of partial orderings, then the lottery sum is the partial ordering $\bigoplus \mathfrak{A}=\{\langle\mathbb{P}, p\rangle: \mathbb{P} \in \mathfrak{A}$ and $p \in \mathbb{P}\} \cup\{0\}$, ordered with 0 below everything and $\langle\mathbb{P}, p\rangle \leq\left\langle\mathbb{P}^{\prime}, p^{\prime}\right\rangle$ iff $\mathbb{P}=\mathbb{P}^{\prime}$ and $p \leq p^{\prime}$. Intuitively, if $G$ is $V$-generic over 
$\mathfrak{A}$, then $G$ first selects an element of $\mathfrak{A}$ (or as Hamkins says in [10], "holds a lottery among the posets in $\left.\mathfrak{A}^{\prime \prime}\right)$ and then forces with it $\left(^{1}\right)$.

A result which will be key in the proof of Theorem 1 is Hamkins' Gap Forcing Theorem of [8] and [9]. We therefore state this theorem now, along with some associated terminology, quoting freely from [8] and [9]. Suppose $\mathbb{P}$ is a partial ordering which can be written as $\mathbb{Q} * \dot{\mathbb{R}}$, where $|\mathbb{Q}|<\delta$ and $\vdash_{\mathbb{Q}}$ " $\mathbb{R}$ is $\delta$-strategically closed". In Hamkins' terminology of [7]-[9], $\mathbb{P}$ admits a gap at $\delta$. Also, as in the terminology of [8] and [9] (and elsewhere), an embedding $j: \bar{V} \rightarrow \bar{M}$ is amenable to $\bar{V}$ when $j\lceil A \in \bar{V}$ for any $A \in \bar{V}$. The Gap Forcing Theorem is then the following.

Theorem 2 (Hamkins' Gap Forcing Theorem). Suppose that $V[G]$ is a forcing extension obtained by forcing that admits a gap at some $\delta<\kappa$ and $j: V[G] \rightarrow M[j(G)]$ is an embedding with critical point $\kappa$ for which $M[j(G)]$ $\subseteq V[G]$ and $M[j(G)]^{\delta} \subseteq M[j(G)]$ in $V[G]$. Then $M \subseteq V$; indeed, $M=$ $V \cap M[j(G)]$. If the full embedding $j$ is amenable to $V[G]$, then the restricted embedding $j\lceil V: V \rightarrow M$ is amenable to $V$. If $j$ is definable from parameters (such as a measure or extender) in $V[G]$, then the restricted embedding $j\lceil V$ is definable from the names of those parameters in $V$.

Finally, we mention that we are assuming familiarity with the large cardinal notions of measurability, strongness, strong compactness, and supercompactness. Interested readers may consult [11] for further details. Also, unlike [11], we will say that the cardinal $\kappa$ is $\lambda$ strong for $\lambda>\kappa$ if there is $j: V \rightarrow M$ an elementary embedding having critical point $\kappa$ so that $j(\kappa)>\left|V_{\lambda}\right|$ and $V_{\lambda} \subseteq M$. As always, $\kappa$ is strong if $\kappa$ is $\lambda$ strong for every $\lambda>\kappa$.

2. The proof of Theorem 1. Let $V_{0} \vDash$ "ZFC $+\kappa_{0}$ is supercompact + There are no cardinals $\delta<\lambda$ such that $\delta$ is $\lambda$ supercompact and $\lambda$ is measurable". Without loss of generality, by first forcing GCH and then following this by the forcing of [4] if necessary, we may also assume that in addition to the preceding, $V_{0} \vDash$ "GCH + Level by level equivalence between strong compactness and supercompactness holds". We then let $\kappa<\kappa_{0}$ be the least strong cardinal in $V_{0}$ (we know $\kappa<\kappa_{0}$ by Lemma 2.1 of [2]), and take $V$ as $V_{0}$ truncated at $\kappa^{*}$, where for the rest of this paper, for $\gamma$ an ordinal, $\gamma^{*}$ is the least cardinal above $\gamma$ which is $2^{\gamma^{*}}$ supercompact. Note that $V \vDash$ " $\kappa$ is the least strong cardinal", since otherwise, if $V \vDash " \delta<\kappa$ is a strong cardinal", then $V_{0} \vDash$ " $\delta$ is $\gamma$ strong for every $\gamma<\kappa$ and $\kappa$ is strong",

$\left({ }^{1}\right)$ The terminology "lottery sum" is due to Hamkins, although the concept of the lottery sum of partial orderings has been around for quite some time and has been referred to at different junctures via the names "disjoint sum of partial orderings", "side-by-side forcing", and "choosing which partial ordering to force with generically". 
so by the proof of Lemma 2.1 of [2], $V_{0} \vDash$ " $\delta<\kappa$ is a strong cardinal", a contradiction.

Note also that by reflection in $V_{0}$ and the supercompactness of $\kappa_{0}$, for any $\gamma<\kappa,\left\{\delta<\kappa_{0}: \delta\right.$ is $\delta^{+\gamma}$ supercompact $\}$ is unbounded in $\kappa_{0}$, meaning that a $\delta$ with this property exists above $\kappa$. Therefore, by further reflection in $V_{0}$ via the strongness of $\kappa,\left\{\delta<\kappa: \delta\right.$ is $\delta^{+\gamma}$ supercompact $\}$ is unbounded in $\kappa$. In fact, this same argument shows that, e.g., $\left\{\delta<\kappa: \delta\right.$ is $\delta^{+\delta}$ supercompact $\}$ is unbounded in $\kappa,\left\{\delta<\kappa: \delta\right.$ is $\delta^{+\delta+1}$ supercompact $\}$ is unbounded in $\kappa$, etc. In any case, for any $\gamma<\kappa$, there is a cardinal $\delta \in[\gamma, \kappa)$ such that $\delta$ is $\delta^{+\gamma}$ supercompact. Also, since level by level equivalence between strong compactness and supercompactness holds in $V_{0}$ and $V \subseteq V_{0}$, level by level equivalence between strong compactness and supercompactness holds in $V$ as well.

Working in $V$ (which is clearly a model for GCH), we define the partial ordering $\mathbb{P}$ used in the proof of Theorem $1 . \mathbb{P}$ is the Easton support iteration of length $\kappa$ which begins by adding a Cohen subset of $\omega$ and then is trivial forcing except at those stages $\delta<\kappa$ which are not themselves $2^{\delta}=\delta^{+}$ supercompact in $V$ but which are in $V$ measurable limits of cardinals $\gamma$ which are $2^{\gamma}=\gamma^{+}$supercompact. At such a stage $\delta$, the forcing done is the lottery sum of all $\delta$-strategically closed partial orderings having rank below $\left(\delta^{*}\right)^{V}$.

\section{Lemma 2.1. $V^{\mathbb{P}} \vDash " \kappa$ is an indestructible strong cardinal".}

Proof. Let $\mathbb{Q} \in V^{\mathbb{P}}$ be such that $V^{\mathbb{P}} \vDash$ "Q⿱Q is $\kappa$-strategically closed". By the definition of $V$, let $\lambda>|\mathrm{TC}(\dot{\mathbb{Q}})|$ be such that $\lambda$ is inaccessible. Fix $j: V \rightarrow M$ an elementary embedding witnessing the $\lambda$ strongness of $\kappa$. Since $V \vDash$ "No cardinal $\gamma>\kappa$ is $2^{\gamma}$ supercompact", $M \vDash$ "No cardinal $\gamma \in(\kappa, \lambda)$ is $2^{\gamma}$ supercompact". Further, since $V \vDash$ " $\kappa$ is the least strong cardinal", by Lemma 2.1 of [2] and the choice of $\lambda, M \vDash$ " $\kappa$ is not $2^{\kappa}$ supercompact". Thus, in $M, \kappa$ is a stage at which a forcing given by a lottery sum is done in $j(\mathbb{P}), \dot{\mathbb{Q}}$ is a term for a partial ordering allowed at the stage $\kappa$ lottery in $j(\mathbb{P})$, and the first ordinal above $\kappa$ which is a non-trivial stage of forcing for $j(\mathbb{P})$ is above $\lambda$ as well.

We show that in $V^{\mathbb{P} * \dot{\mathbb{Q}}}, j$ lifts to a $\lambda$ strong embedding $j: V^{\mathbb{P} * \dot{\mathbb{Q}}} \rightarrow$ $M^{j(\mathbb{P} * \dot{\mathbb{Q}})}$. This will complete the proof of Lemma 2.1, since $\lambda$ may be chosen arbitrarily large in the universe.

The argument that the embedding $j$ lifts is quite similar to the argument given in the proof of Theorem 4.10 of [10], or the argument given in the proof of Lemma 4.2 of [1]. For the benefit of readers, we give the argument here as well, again taking the liberty to quote freely from the proofs of Theorem 4.10 of [10] and Lemma 4.2 of [1]. We may assume that $M=\left\{j(f)(a): a \in[\lambda]^{<\omega}\right.$, $f \in V$, and $\left.\operatorname{dom}(f)=[\kappa]^{|a|}\right\}$. Since we may write $j(\mathbb{P})$ as $\mathbb{P} * \dot{\mathbb{Q}} * \dot{\mathbb{R}}$, by our 
remarks above, we know that the first ordinal at which $\dot{\mathbb{R}}$ is forced to do a lottery sum forcing is above $\lambda$. Since $\lambda$ is inaccessible, we have $M^{\kappa} \subseteq M$. This means that if $G$ is $V$-generic over $\mathbb{P}$ and $H$ is $V[G]$-generic over $\mathbb{Q}$, then $\mathbb{R}$ is $\prec \kappa^{+}$-strategically closed in both $V[G][H]$ and $M[G][H]$, and $\mathbb{R}$ is $\lambda$-strategically closed in $M[G][H]$.

As in [10] and [1], by using a suitable coding that allows us to identify finite subsets of $\lambda$ with elements of $\lambda$, by the definition of $M$, there must be some $\alpha<\lambda$ and function $g$ such that $\dot{\mathbb{Q}}=j(g)(\alpha)$. Let $N=\left\{i_{G * H}(\dot{z})\right.$ : $\dot{z}=j(f)(\kappa, \alpha, \lambda)$ for some function $f \in V\}$. It is easy to verify that $N \prec$ $M[G][H]$, that $N$ is closed under $\kappa$ sequences in $V[G][H]$, and that $\kappa, \alpha, \lambda$, $\mathbb{Q}$, and $\mathbb{R}$ are all elements of $N$. Further, since $\mathbb{R}$ is $j(\kappa)$-c.c. in $M[G][H]$ and there are only $2^{\kappa}=\kappa^{+}$many functions $f: \kappa \rightarrow V_{\kappa}$ in $V$, there are at most $\kappa^{+}$ many dense open subsets of $\mathbb{R}$ in $N$. Therefore, since $\mathbb{R}$ is $\prec \kappa^{+}$-strategically closed in both $M[G][H]$ and $V[G][H]$, we can build $H^{\prime} \subseteq \mathbb{R}$ in $V[G][H]$ as follows. Let $\left\langle D_{\sigma}: \sigma<\kappa^{+}\right\rangle$enumerate in $V[G][H]$ the dense open subsets of $\mathbb{R}$ present in $N$ so that every dense open subset of $\mathbb{R}$ occurring in $N$ appears at an odd stage at least once in the enumeration. If $\sigma$ is an odd ordinal, $\sigma=\tau+1$ for some $\tau$. Player I picks $p_{\sigma} \in D_{\sigma}$ extending $q_{\tau}$ (initially, $q_{0}$ is the empty condition), and player II responds by picking $q_{\sigma} \geq p_{\sigma}$ according to a fixed strategy $\mathcal{S}$ (so $q_{\sigma} \in D_{\sigma}$ ). If $\sigma$ is a limit ordinal, player II uses $\mathcal{S}$ to pick $q_{\sigma}$ extending each $q \in\left\langle q_{\beta}: \beta<\sigma\right\rangle$. By the $\prec \kappa^{+}$-strategic closure of $\mathbb{R}$ in $V[G][H]$, player II's strategy can be assumed to be a winning one, so $\left\langle q_{\sigma}: \sigma<\kappa^{+}\right\rangle$can be taken as an increasing sequence of conditions with $q_{\sigma} \in D_{\sigma}$ for $\sigma<\kappa^{+}$.

Let $H^{\prime}=\left\{p \in \mathbb{R}: \exists \sigma<\kappa^{+}\left[q_{\sigma} \geq p\right]\right\}$. We show now that $H^{\prime}$ is actually $M[G][H]$-generic over $\mathbb{R}$. If $D$ is a dense open subset of $\mathbb{R}$ in $M[G][H]$, then $D=i_{G * H}(\dot{D})$ for some name $\dot{D} \in M$. Consequently, $\dot{D}=j(f)\left(\kappa, \kappa_{1}, \ldots, \kappa_{n}\right)$ for some function $f \in V$ and $\kappa<\kappa_{1}<\ldots<\kappa_{n}<\lambda$. Let $\bar{D}$ be a name for the intersection of all $i_{G * H}\left(j(f)\left(\kappa, \alpha_{1}, \ldots, \alpha_{n}\right)\right)$, where $\kappa<\alpha_{1}<\ldots<\alpha_{n}<\lambda$ is such that $j(f)\left(\kappa, \alpha_{1}, \ldots, \alpha_{n}\right)$ yields a name for a dense open subset of $\mathbb{R}$. Since this name can be given in $M$ and $\mathbb{R}$ is $\lambda$-strategically closed in $M[G][H]$ and therefore $\lambda$-distributive in $M[G][H], \bar{D}$ is a name for a dense open subset of $\mathbb{R}$ which is definable without the parameters $\kappa_{1}, \ldots, \kappa_{n}$. Hence, by its definition, $i_{G * H}(\bar{D}) \in N$. Thus, since $H^{\prime}$ meets every dense open subset of $\mathbb{R}$ present in $N, H^{\prime} \cap i_{G * H}(\bar{D}) \neq \emptyset$, so since $\bar{D}$ is forced to be a subset of $\dot{D}$, $H^{\prime} \cap i_{G * H}(\dot{D}) \neq \emptyset$. This means $H^{\prime}$ is $M[G][H]$-generic over $\mathbb{R}$, so in $V[G][H]$, as $j^{\prime \prime} G \subseteq G * H * H^{\prime}, j$ lifts to $j: V[G] \rightarrow M[G][H]\left[H^{\prime}\right]$ via the definition $j\left(i_{G}(\tau)\right)=i_{G * H * H^{\prime}}(j(\tau))$.

It remains to lift $j$ through the forcing $\mathbb{Q}$ while working in $V[G][H]$. To do this, it suffices to show that $j^{\prime \prime} H \subseteq j(\mathbb{Q})$ generates an $M[G][H]\left[H^{\prime}\right]$ generic object $H^{\prime \prime}$ over $j(\mathbb{Q})$. Given a dense open subset $D \subseteq j(\mathbb{Q})$ with 
$D \in M[G][H]\left[H^{\prime}\right], D=i_{G * H * H^{\prime}}(\dot{D})$ for some name $\dot{D}=j(\vec{D})(a)$, where $a \in[\lambda]^{<\omega}$ and $\vec{D}=\left\langle D_{\sigma}: \sigma \in[\kappa]^{|a|}\right\rangle$ is a function. We may assume that every $D_{\sigma}$ is a dense open subset of $\mathbb{Q}$. Since $\mathbb{Q}$ is $\kappa$-distributive, it follows that $D^{\prime}=\bigcap_{\sigma \in[\kappa]^{|a|}} D_{\sigma}$ is also a dense open subset of $\mathbb{Q}$. As $j\left(D^{\prime}\right) \subseteq D$ and $H \cap D^{\prime} \neq \emptyset, j^{\prime \prime} H \cap D \neq \emptyset$. Thus, $H^{\prime \prime}=\left\{p \in j(\mathbb{Q}): \exists q \in j^{\prime \prime} H[q \geq p]\right\}$ is our desired generic object, and $j$ lifts to $j: V[G][H] \rightarrow M[G][H]\left[H^{\prime}\right]\left[H^{\prime \prime}\right]$. This final lifted version of $j$ is $\lambda$ strong since $V_{\lambda} \subseteq M$, meaning $\left(V_{\lambda}\right)^{V[G][H]} \subseteq$ $M[G][H] \subseteq M[G][H]\left[H^{\prime}\right]\left[H^{\prime \prime}\right]$. This completes the proof of Lemma 2.1.

Lemma 2.2. If $\lambda>\delta$ is a cardinal, then $V \vDash$ " $\delta$ is $\lambda$ supercompact" iff $V^{\mathbb{P}} \vDash " \delta$ is $\lambda$ supercompact".

Proof. Suppose $\lambda>\delta$. Assume first that $V^{\mathbb{P}} \vDash$ " $\delta$ is $\lambda$ supercompact". Write $\mathbb{P}=\mathbb{P}_{0} * \dot{\mathbb{P}}_{1}$, where $\left|\mathbb{P}_{0}\right|=\omega$ and $\vdash_{\mathbb{P}_{0}}$ " $\dot{\mathbb{P}}_{1}$ is $\aleph_{1}$-strategically closed". Since $\mathbb{P}$ admits a gap at $\aleph_{1}$, by the Gap Forcing Theorem of [8] and [9], $V \vDash$ " $\delta$ is $\lambda$ supercompact" as well. In fact, the Gap Forcing Theorem of [8] and [9] implies that for any $\gamma \geq \delta$ (cardinal or ordinal) such that $V^{\mathbb{P}} \vDash$ " $\delta$ is $\gamma$ supercompact", $V \vDash$ " $\delta$ is $\gamma$ supercompact".

Assume now that $V \vDash$ " $\delta$ is $\lambda$ supercompact" and $\lambda$ is regular. By our assumptions on $V$ and $V_{0}, \delta<\kappa$ and $\lambda<\eta$, where for the remainder of the proof of Lemma 2.2, $\eta$ is the least $V$-measurable cardinal above $\delta$.

Let $A$ be the set of non-trivial stages of forcing below $\delta$, and let $\mathbb{P}_{A}$ be the portion of $\mathbb{P}$ defined using the elements of $A$. Rewrite $\mathbb{P}=\mathbb{P}_{A} * \dot{\mathbb{Q}}$. We begin by showing that $V^{\mathbb{P}_{A}} \vDash$ " $\delta$ is $\lambda$ supercompact". If $A$ is bounded below $\delta$, then if $A$ contains a largest element $\gamma$, since $\lambda>\delta$ (meaning that $\delta$ is at least $2^{\delta}=\delta^{+}$supercompact), $\gamma^{*} \leq \delta$. By the definition of the lottery sum done at stage $\gamma$, the forcing selected must have cardinality below $\gamma^{*}$. This means that $\mathbb{P}_{A}$ is forcing equivalent to a partial ordering having size below $\delta$, so by the Lévy-Solovay results [14], $V^{\mathbb{P}_{A}} \vDash$ " $\delta$ is $\lambda$ supercompact". If, however, $A$ is bounded below $\delta$ and does not contain a largest element, then by its definition, $\left|\mathbb{P}_{A}\right|<\delta$. Hence, once again, the results of [14] tell us that $V^{\mathbb{P}_{A}} \vDash$ " $\delta$ is $\lambda$ supercompact".

We may therefore assume that $A$ is unbounded in $\lambda$. Let $j: V \rightarrow M$ be an elementary embedding witnessing the $\lambda$ supercompactness of $\delta$ generated by a normal ultrafilter over $P_{\delta}(\lambda)$ such that $M \vDash$ " $\delta$ is not $\lambda$ supercompact". Write $j\left(\mathbb{P}_{A}\right)=\mathbb{P}_{A} * \dot{\mathbb{R}}$, and let $G$ be $V$-generic over $\mathbb{P}_{A}$. Since $\mathbb{P}_{A}$ is an Easton support iteration and therefore satisfies $\delta$-c.c., standard arguments show that $M[G]$ remains $\lambda$ closed with respect to $V[G]$. Further, by opting for trivial forcing at stage $\delta$ in $M$ if necessary, we may infer that $\vdash_{\mathbb{P}_{A}}$ "The first ordinal which is a non-trivial stage of forcing for $\mathbb{\mathbb { R }}$ above $\delta$ is above $\lambda$ ". This is since $\lambda<\eta, M^{\lambda} \subseteq M, M \vDash$ " $\delta$ is not $\lambda$ supercompact", and every non-trivial stage of forcing in $M$ must occur at an $M$-measurable cardinal. We may therefore assume without loss of generality that $\Vdash_{\mathbb{P}_{A}}$ " $\mathbb{R}$ is $\lambda$-strategically closed". In 
addition, the definition of $\mathbb{P}_{A}$ tells us that $\vdash_{\mathbb{P}_{A}}$ "| $|\dot{\mathbb{R}}|=j(\delta)$ ", meaning by GCH in both $V$ and $M$ that the number of dense open subsets of $\mathbb{R}$ in $M[G]$ is at most $2^{j(\delta)}=j(\delta)^{+}=j\left(\delta^{+}\right)$. Since $M$ is generated via an ultrapower and $\lambda$ is regular, by GCH in $V,\left|[\lambda]^{<\delta}\right|=\lambda$ and $\left|j\left(\delta^{+}\right)\right|=\mid\left\{f: f: P_{\delta}(\lambda) \rightarrow \delta^{+}\right.$ is a function $\}|=|\left\{f: f: \lambda \rightarrow \delta^{+}\right.$is a function $\} \mid=2^{\lambda}=\lambda^{+}$. This means we can use the argument given in Lemma 2.1 for the construction of the generic object $H^{\prime}$ to construct in $V[G]$ an $M[G]$-generic object $H$ over $\mathbb{R}$ such that $j^{\prime \prime} G \subseteq G * H$ and lift in the usual way $j$ to $j: V[G] \rightarrow M[G][H]$ via the definition $j\left(i_{G}(\tau)\right)=i_{G * H}(j(\tau))$. Hence, it is once again true that $V^{\mathbb{P}_{A}} \vDash$ " $\delta$ is $\lambda$ supercompact".

Observe that $\delta$ cannot be a stage at which a lottery sum forcing is done, since such a $\delta$ is not $2^{\delta}=\delta^{+}$supercompact in $V$, which is impossible because $\lambda>\delta$ and $V \vDash$ " $\delta$ is $\lambda$ supercompact". Therefore, as $\delta$ is a stage at which no lottery sum forcing is done (meaning that only trivial forcing is done at stage $\delta$ ), by the definition of $\mathbb{P}, \vdash_{\mathbb{P}_{A}}$ "Q⿱亠乂 is $\eta$-strategically closed". Since we have already observed that $\lambda<\eta$, we are now able to infer that $V^{\mathbb{P}_{A} * \dot{\mathbb{Q}}}=V^{\mathbb{P}} \vDash$ " $\delta$ is $\lambda$ supercompact".

We complete the proof of Lemma 2.2 by noting what happens when $V \vDash$ " $\delta$ is $\lambda$ supercompact" and $\lambda$ is singular. If this is the case, then if $\lambda$ has cofinality at least $\delta$, since GCH in $V$ tells us that $\left|[\lambda]^{<\delta}\right|=\lambda$ and $\left|j\left(\delta^{+}\right)\right|=\lambda^{+}$, the same proof as just given immediately shows that $V^{\mathbb{P}_{A}} \vDash$ " $\delta$ is $\lambda$ supercompact". If the cofinality of $\lambda$ is below $\delta$, then by GCH in $V, V \vDash$ " $\delta$ is $\lambda^{+}$supercompact" (see the introductory section of [4] for a discussion of this fact). We therefore once again see that $V^{\mathbb{P}_{A}} \vDash$ " $\delta$ is both $\lambda^{+}$ and $\lambda$ supercompact". Since $\vdash_{\mathbb{P}_{A}}$ "这 is $\eta$-strategically closed", this means that $V^{\mathbb{P}_{A} * \dot{\mathbb{Q}}}=V^{\mathbb{P}} \vDash$ " $\delta$ is $\lambda$ supercompact", regardless of our cofinality assumptions on $\lambda$. This completes the proof of Lemma 2.2.

LEMma 2.3. $V^{\mathbb{P}} \vDash$ "Level by level equivalence between strong compactness and supercompactness holds below $\kappa$ ".

Proof. We begin by noting that the case $\delta=\lambda$ is immediate, since for any cardinal $\sigma$ and any ordinal $\varrho$ having cardinality $\sigma$ (in particular, $\varrho=\sigma$ ), $\sigma$ is measurable iff $\sigma$ is $\varrho$ strongly compact iff $\sigma$ is $\varrho$ supercompact. We may therefore fix $\delta<\lambda<\kappa$ such that $\delta$ and $\lambda$ are both regular cardinals and $V^{\mathbb{P}} \vDash$ " $\delta$ is $\lambda$ strongly compact". It is then automatic that $V^{\mathbb{P}} \vDash$ " $\delta$ is measurable", and by the argument given in the first paragraph of the proof of Lemma 2.2, $V \vDash$ " $\delta$ is measurable" as well.

We begin by assuming that for no non-trivial stage of forcing $\gamma$ do we have $\delta \in\left(\gamma, \gamma^{*}\right)$. Note that under these circumstances, $\mathbb{P}$ is "mild" with respect to $\delta$, where as in [8] and [9], a partial ordering $\mathbb{Q}$ is mild with respect to a cardinal $\gamma$ if every set of ordinals $x$ in $V^{\mathbb{Q}}$ of size below $\gamma$ has a "nice" name $\tau$ in $V$ of size below $\gamma$, i.e., there is a set $y$ in $V,|y|<\gamma$, such that 
any ordinal forced by a condition in $\mathbb{Q}$ to be in $\tau$ is an element of $y$. To see this, as in the proof of Lemma 2.2, let $A$ be the set of non-trivial stages of forcing below $\delta$, and let $\mathbb{P}_{A}$ be the portion of $\mathbb{P}$ defined using the elements of $A$. Rewrite $\mathbb{P}=\mathbb{P}_{A} * \dot{\mathbb{Q}}$. Since $\delta$ is measurable in $V$, by the definition of $\mathbb{P}, \Vdash_{\mathbb{P}_{A}}$ "⿺辶Q is (at least) $\delta$-strategically closed". Hence, to show that $\mathbb{P}$ is mild with respect to $\delta$, it suffices to show that $\mathbb{P}_{A}$ is mild with respect to $\delta$.

If $A$ contains a largest element $\gamma$, then by the definition of the lottery sum done at stage $\gamma$, the forcing selected must have cardinality below $\gamma^{*}$. Since $\delta \notin\left(\gamma, \gamma^{*}\right), \gamma^{*} \leq \delta$. This immediately implies that $\mathbb{P}_{A}$ is mild with respect to $\delta$. If $A$ does not contain a largest element, then if $A$ is unbounded in $\delta$, the definition of $\mathbb{P}_{A}$ as an Easton support iteration of strategically closed forcing immediately shows that $\mathbb{P}_{A}$ is mild with respect to $\delta$. If $A$ does not contain a largest element, then if $A$ is bounded in $\delta$, the definition of $\mathbb{P}_{A}$ is such that $\left|\mathbb{P}_{A}\right|<\delta$. Thus, regardless of the exact nature of $A, \mathbb{P}_{A}$ is mild with respect to $\delta$, so $\mathbb{P}$ is mild with respect to $\delta$. Therefore, by Corollary 7 of [8] and Corollary 16 of [9], which tell us that a partial ordering admitting a gap at $\aleph_{1}$ which is mild with respect to a cardinal $\varrho$ creates no new instances of strong compactness for $\varrho$, it must be the case that $V \vDash$ " $\delta$ is $\lambda$ strongly compact". By our assumptions on $V_{0}$ and $V$, we immediately see $V \vDash$ " $\delta$ is $\lambda$ supercompact" and that $\lambda$ is below the least $V$-measurable cardinal above $\delta$. By Lemma $2.2, V^{\mathbb{P}} \vDash$ " $\delta$ is $\lambda$ supercompact".

We next handle what happens when there is a non-trivial stage of forcing $\gamma$ for which $\delta \in\left(\gamma, \gamma^{*}\right)$. We show that when this holds, $V^{\mathbb{P}} \vDash$ " $\delta$ is not $\delta^{+}$ strongly compact". By the remarks given in the first paragraph of the proof of this lemma, this completes the proof of Lemma 2.3.

Note that under these circumstances, since $\gamma$ is not $2^{\gamma}$ supercompact in $V$ and $\gamma^{*}$ is the least cardinal above $\gamma$ which is $2^{\gamma^{*}}$ supercompact in $V, \delta$ is not $2^{\delta}=\delta^{+}$supercompact in $V$. This means that in $V$, using level by level equivalence, $\delta$ is not $\delta^{+}$strongly compact.

We now assume $V^{\mathbb{P}} \vDash$ " $\delta$ is $\delta^{+}$strongly compact" and consider the following two cases.

CASE 1: $\left(\delta^{+}\right)^{V}<\left(\delta^{+}\right)^{V^{\mathbb{P}}}$. If this is the situation, then as $\delta$ is measurable and hence a cardinal in $V^{\mathbb{P}}, V^{\mathbb{P}} \vDash "\left|\left(\delta^{+}\right)^{V}\right|=\delta$ ". Therefore, by our remarks above, $V^{\mathbb{P}} \vDash$ " $\delta$ is $\left(\delta^{+}\right)^{V}$ supercompact". By the Gap Forcing Theorem of [8] and [9] (see also Theorem 5.3 of [10]), $V \vDash$ " $\delta$ is $\left(\delta^{+}\right)^{V}=\delta^{+}$supercompact", an immediate contradiction.

CASE 2: $\left(\delta^{+}\right)^{V}=\left(\delta^{+}\right)^{V^{\mathbb{P}}}$. To handle when this occurs, we use a new idea due to Hamkins, which will appear in [6] in a more general context. Hamkins' argument is as follows. Let $G$ be $V$-generic over $\mathbb{P}$, and let $j: V[G] \rightarrow$ $M[j(G)]$ be an elementary embedding witnessing the $\delta^{+}$strong compactness of $\delta$ generated by a $\delta$-additive, fine ultrafilter over $P_{\delta}\left(\delta^{+}\right)$present in $V[G]$. 
As $\mathbb{P}$ admits a gap at $\aleph_{1}$ and $M[j(G)]^{\delta} \subseteq M[j(G)]$, by the Gap Forcing Theorem of [8] and [9], the embedding $j^{*}=j\lceil V: V \rightarrow M$ is definable in $V$. Note that $j$ and $j^{*}$ agree on the ordinals. Since $j$ is a $\delta^{+}$strong compactness embedding in $V[G]$, there is some $X \subseteq j\left(\delta^{+}\right), X \in M[j(G)]$ with $j^{\prime \prime} \delta^{+} \subseteq X$ and $M[j(G)] \vDash "|X|<j\left(\delta^{+}\right)$". Therefore, since $\delta^{+}$is regular in $V[G], j\left(\delta^{+}\right)$is regular in $M[j(G)]$, so we can find an $\alpha<j\left(\delta^{+}\right)$with $\alpha>$ $\sup (X) \geq \sup \left(j^{\prime \prime} \delta^{+}\right)$. This means that if $x \subseteq \delta^{+}$is such that $x \subseteq \beta<\delta^{+}$, $j(\alpha) \notin j(x) \subseteq j(\beta)$. But then $\mathcal{U}=\left\{x \subseteq \delta^{+}: \alpha \in j^{*}(x)\right\}$ defines in $V$ a $\delta$-additive, uniform ultrafilter over $\delta^{+}$which gives measure 1 to sets having size $\delta^{+}$. By a theorem of Ketonen [12], $\delta$ is $\delta^{+}$strongly compact in $V$, a contradiction.

Thus, assuming that $V^{\mathbb{P}} \vDash$ " $\delta$ is $\delta^{+}$strongly compact" leads to absurdities. Therefore, Cases 1 and 2 complete the proof of Lemma 2.3.

We observe that in the construction of $V^{\mathbb{P}}$, there will be non-trivial stages of forcing $\delta$ at which measurable cardinals between $\delta$ and $\left(\delta^{*}\right)^{V}$ are destroyed. These will occur, e.g., whenever the lottery at stage $\delta$ opts for the appropriate Lévy collapse. We thus cannot infer in general that $V \vDash$ " $\delta$ is $\lambda$ supercompact" iff $V^{\mathbb{P}} \vDash$ " $\delta$ is $\lambda$ supercompact". However, Lemma 2.2 shows that this is indeed the situation if $\lambda>\delta$.

Lemma 2.4. $V^{\mathbb{P}} \vDash$ "For any $\gamma<\kappa$, there is a cardinal $\delta \in[\gamma, \kappa)$ such that $\delta$ is $\delta^{+\gamma}$ supercompact".

Proof. By our discussion at the beginning of the proof of Theorem 1, for any $\gamma<\kappa$, we can find in $V$ a cardinal $\delta \in[\gamma, \kappa)$ such that $\delta$ is $\delta^{+\gamma}$ supercompact. By Lemma $2.2, V^{\mathbb{P}} \vDash$ " $\delta$ is $\delta^{+\gamma}$ supercompact". This completes the proof of Lemma 2.4 .

Since $\mathbb{P}$ can be defined so that $|\mathbb{P}|=\kappa$, by the results of [14], $V^{\mathbb{P}} \vDash$ "No cardinal $\lambda>\kappa$ is $2^{\lambda}$ supercompact + Level by level equivalence between strong compactness and supercompactness holds above $\kappa$ ". As $V^{\mathbb{P}} \vDash$ " $\kappa$ is a strong cardinal", any failure of level by level equivalence between strong compactness and supercompactness at $\kappa$ would have to be reflected below $\kappa$, contradicting Lemma 2.3. These remarks, together with Lemmas 2.1-2.4, complete the proof of Theorem 1.

We observe that by the Gap Forcing Theorem of [8] and [9], since $\kappa$ is the least strong cardinal in $V, \kappa$ is the least strong cardinal in $V^{\mathbb{P}}$ as well.

3. Concluding remarks. We conclude this paper by noting that by assuming our initial model $V_{0}$ contains a supercompact cardinal, we are able to ensure that the large cardinal structure in terms of supercompactness below our indestructible strong cardinal is non-trivial. Indeed, if the supercompact cardinal $\kappa_{0}$ has large cardinals such as inaccessibles, Mahlos, weakly 
compacts, Ramseys, etc. above it, then our methods of proof show that in $V_{0}, V$, and consequently in $V^{\mathbb{P}}$, for any $\gamma<\kappa$, there are cardinals $\delta<\lambda$, $\delta, \lambda \in(\gamma, \kappa)$ such that $\delta$ is $\lambda$ supercompact and $\lambda$ is inaccessible, Mahlo, weakly compact, Ramsey, etc. It is possible, however, to force over a canonical inner model for a strong cardinal $\kappa$ with, e.g., the partial ordering of Theorem 4.10 of [10] to obtain a model in which $\kappa$ is an indestructible strong cardinal. In this model, no cardinal $\delta$ is $\delta^{+}$strongly compact, and level by level equivalence between strong compactness and supercompactness holds trivially.

\section{References}

[1] A. Apter, Some remarks on indestructibility and Hamkins' lottery preparation, Arch. Math. Logic, to appear.

[2] A. Apter and J. Cummings, Identity crises and strong compactness II: strong cardinals, Arch. Math. Logic 40 (2001), 25-38.

[3] A. Apter and J. D. Hamkins, Indestructibility and the level-by-level agreement between strong compactness and supercompactness, J. Symbolic Logic 67 (2002), 820840 .

[4] A. Apter and S. Shelah, On the strong equality between supercompactness and strong compactness, Trans. Amer. Math. Soc. 349 (1997), 103-128.

[5] M. Gitik and S. Shelah, On certain indestructibility of strong cardinals and a question of Hajnal, Arch. Math. Logic 28 (1989), 35-42.

[6] J. D. Hamkins, A generalized version of the Gap Forcing Theorem, in preparation.

[7] —, Destruction or preservation as you like it, Ann. Pure Appl. Logic 91 (1998), 191-229.

[8] —, Gap forcing, Israel J. Math. 125 (2001), 237-252.

[9] —, Gap forcing: generalizing the Lévy-Solovay theorem, Bull. Symbolic Logic 5 (1999), 264-272.

[10] -, The lottery preparation, Ann. Pure Appl. Logic 101 (2000), 103-146.

[11] A. Kanamori, The Higher Infinite, Springer, Berlin, 1994.

[12] J. Ketonen, Strong compactness and other cardinal sins, Ann. Math. Logic 5 (1972), $47-76$.

[13] R. Laver, Making the supercompactness of $\kappa$ indestructible under $\kappa$-directed closed forcing, Israel J. Math. 29 (1978), 385-388.

[14] A. Lévy and R. Solovay, Measurable cardinals and the continuum hypothesis, Israel J. Math. 5 (1967), 234-248.

Department of Mathematics

Baruch College of CUNY

New York, NY 10010, U.S.A.

E-mail: awabb@cunyvm.cuny.edu

Web: http://math.baruch.cuny.edu/ ${ }^{\sim}$ apter

Received 30 June 2002;

in revised form 5 February 2003 